\title{
Avaliação do ácido trans, trans-mucônico urinário como biomarcador de exposição ao benzeno
}

\section{Assessment of urinary trans, trans-muconic acid as a biomarker of exposure to benzene}

\author{
Flávia Cristina S de Paula, Josiane Nicácio Silveira, Roberto Gonçalves Junqueira e Edna \\ Maria Alvarez Leite
}

Departamento de Análises Clínicas e Toxicológicas da Faculdade de Farmácia da Universidade Federal de Minas Gerais. Belo Horizonte, MG, Brasil

\section{Descritores}

Benzeno, análise. Exposição ocupacional. Marcadores biológicos. Ácido trans, trans-mucônico.

\section{Keywords}

Benzene, analysis. Occupational exposure. Biological markers. Trans, trans-muconic acid.

\section{Resumo}

\section{Objetivo}

Avaliar o uso do ácido trans, trans-mucônico urinário como biomarcador na monitorização da exposição ocupacional ao benzeno.

\section{Métodos}

Foi estudado o comportamento do ácido trans, trans-mucônico em amostras de urina de indivíduos expostos $(\mathrm{N}=36)$ e não expostos $(\mathrm{N}=116)$ ocupacionalmente ao solvente. A concentração urinária do ácido foi determinada por Cromatografia Líquida de Alta Eficiência. A amostra foi constituída de indivíduos expostos ao benzeno em uma refinaria de petróleo localizada em Belo Horizonte, MG. Foram empregados os testes estatísticos não-paramétricos de Kruskall-Wallis, Mann Witney e de correlação de Spearman, ao nível de significância de $0,05 \%$.

\section{Resultados}

A exposição média ao benzeno dos trabalhadores selecionados foi de $0,15 \pm 0,05 \mathrm{mg} /$ $\mathrm{m}^{3}(0,05 \mathrm{ppm})$ o que resultou em um valor médio do metabólito urinário de $0,19 \pm 0,04$ $\mathrm{mg} / \mathrm{g}$ de creatinina. A faixa de referência do ácido trans, trans-mucônico no grupo não exposto variou de 0,03 a $0,26 \mathrm{mg} / \mathrm{g}$ de creatinina (média de $0,10 \pm 0,08 \mathrm{mg} / \mathrm{g}$ de creatinina). Foi encontrada uma diferença significativa entre os níveis de ácido trans, trans-mucônico do grupo exposto e não exposto. Entretanto, não houve correlação entre os níveis do metabólito urinário e do benzeno no ar. Foi observada a correlação entre ácido trans, trans-mucônico e hábito de fumar no grupo de indivíduos expostos. A ingestão de álcool num período de até 48 horas antes da coleta das amostras não mostrou interferir nos níveis do metabólito nos dois grupos estudados. Foi observada a correlação entre ácido trans, trans-mucônico e idade (faixa etária de 18 a 25 anos) no grupo de não expostos.

Conclusões

Os resultados obtidos evidenciaram a importância de ser mais bem avaliada a influência do hábito de fumar e da faixa etária do trabalhador nos níveis urinários do ácido trans, trans-mucônico.

\section{Abstract}

\section{Objective}

To assess the use of trans, trans-muconic acid as a biomarker of occupational exposure to benzene.

Baseado na dissertação de mestrado apresentada à Faculdade de Farmácia da Universidade Federal de Minas Gerais, 2001.

Flávia Cristina S. de Paula

Rua D, 40 Betânia

30590-060 Belo Horizonte, MG, Brasil

Subvencionado pela Fundação de Amparo à Pesquisa do Estado de Minas Gerais (FAPEMIG - Processo n. CBS200095) Recebido em 14/4/2002. Reapresentado em 16/4/2003. Aprovado em 30/6/2003. 


\begin{abstract}
Methods
Trans, trans-muconic acid in urine samples of exposed (exposed group, $n=36$ ) and non-exposed (non-exposed group, $n=116$ ) workers to benzene. Urinary levels of trans, trans-muconic acid were quantified by high-performance liquid chromatography. The study sample consisted of subjects exposed to benzene in an oil refinery in Belo Horizonte, Brazil. Non-parametric statistical analysis was carried out using KruskallWallis test, Mann-Whitney test and Spearman correlation at $p<0.05$.

Results

Workers were exposed on average to benzene levels of $0.15 \pm 0.05 \mathrm{mg} / \mathrm{m}^{3}(0.05 \mathrm{ppm})$ and they showed a urinary trans, trans-muconic acid mean value of $0.19 \pm 0.04 \mathrm{mg} / \mathrm{g}$ of creatinine. The reference value range of trans, trans-muconic acid in non-exposed subjects was 0.03 to $0.26 \mathrm{mg} / \mathrm{g}$ of creatinine (mean $0.10 \pm 0.08 \mathrm{mg} / \mathrm{g}$ de creatinine). There was seen a statistical difference between trans, trans-muconic acid levels in urine samples from exposed and non-exposed groups. There was no correlation between urinary trans, trans-muconic acid and air benzene levels. There was no correlation between urinary trans, trans-muconic acid levels in the exposed group and smoking. Alcohol consumption up to 48 hours before sampling procedure showed no effect on trans, trans-muconic acid levels in both exposed and non-exposed groups. There was however a correlation between age (range 18 to 25 years) and urinary metabolite levels in the latter group.
\end{abstract}

Conclusions

The results show that it is important to evaluate the effect of age and smoking habits on urinary trans, trans-muconic acid levels.

\section{INTRODUÇÃO}

As características carcinogênicas do benzeno desencadearam um esforço mundial em restringir, ao máximo, o limite de exposição ao composto. Esta diminuição, entretanto, não assegura a proteção à saúde dos indivíduos expostos, uma vez que não existe um limite seguro de exposição para substâncias cancerígenas. ${ }^{7}$

Diante da relevância da questão, nos últimos anos, uma série de estudos vem sendo desenvolvida, objetivando avaliar e adotar novos biomarcadores e novas metodologias analíticas que apresentem sensibilidade suficiente para permitir a imprescindível avaliação da exposição ocupacional a baixas concentrações de benzeno. ${ }^{10,13}$

No Brasil, o ácido trans, trans-mucônico (ATTM) urinário tem sido o mais estudado. A correlação entre ATTM e benzeno é detectada em níveis ambientais do solvente inferiores a $1,0 \mathrm{ppm},{ }^{2,16}$ fato que o viabiliza como biomarcador no Brasil, considerando que a concentração de benzeno permitida no ar ocupacional (VRT) varia de 1,0 a 2,5 ppm. ${ }^{7}$ Dentre as vantagens do uso desse biomarcador, destacam-se a sensibilidade e a simplicidade analítica de sua determinação urinária. ${ }^{3,5}$ A principal desvantagem do uso deste metabólito como indicador biológico de exposição é o fato de sua concentra- ção basal ser influenciada por fatores e características individuais. ${ }^{15}$

O presente trabalho teve como objetivo avaliar a influência de fatores individuais como sexo, idade, hábito de fumar, dentre outros, sobre a excreção urinária de ATTM em grupos de trabalhadores expostos e não expostos ocupacionalmente ao solvente; e avaliar a correlação do ATTM urinário no grupo exposto com os níveis de benzeno no ar ocupacional e do ATTM nas amostras de urina dos dois grupos de indivíduos selecionados para o estudo.

\section{MÉTODOS}

Foram coletadas 37 amostras de urina de indivíduos expostos ao benzeno em uma refinaria de petróleo da região metropolitana de Belo Horizonte no Estado de Minas Gerais. O nível de exposição ocupacional ao solvente de cada trabalhador foi determinado com o auxílio de monitores passivos individuais para compostos orgânicos voláteis (OVM3500) dispostos ao nível da zona respiratória dos trabalhadores no dia da coleta da amostra biológica. Os indivíduos selecionados encontram-se na faixa etária de 18 a 50 anos $(34,8 \pm 8,7)$, sendo 27 do sexo masculino e 10 do sexo feminino.

Para determinar a concentração urinária de ATTM no grupo não exposto ocupacionalmente ao benzeno, 
Tabela 1 - Caracterização dos indivíduos expostos e não expostos em função das variáveis estudadas.

\begin{tabular}{lcc}
\hline Parâmetros & Grupo não exposto & Grupo exposto \\
\hline Idade média (global) & 30,7 & 34,8 \\
Masculino & 30,1 & 37,8 \\
Feminino & 31,4 & 26,5 \\
Cigarro & $(10,1 \pm 5,5)$ & $13(10,5 \pm 8.2)$ \\
Sim (no cigarro/dia) & 94 & 24 \\
Não & & 29 \\
Álcool & 75 & 8 \\
Sim & 41 & \\
Não & & $27(3,0 \pm 1,9)$ \\
Café & $(3,2 \pm 2,6)$ & 10 \\
Sim (no xícaras/dia) & 95 \\
Não &
\end{tabular}

foram coletadas 116 amostras de indivíduos também residentes na região metropolitana de Belo Horizonte, na faixa etária de 18 a 60 anos $(30,7 \pm 8,3)$, sendo 71 do sexo masculino e 45 do sexo feminino. $\mathrm{Na}$ Tabela 1 estão as características individuais dos grupos selecionados para o trabalho.

Os indivíduos selecionados expostos e não expostos ao benzeno responderam a um protocolo toxicológico no momento da coleta das amostras, fornecendo informações referentes a hábitos e características individuais como idade, sexo, uso de cigarro, consumo de bebidas alcoólicas, entre outras. Todas as amostras de urina foram coletadas em frascos de plástico e transportadas para o laboratório em recipientes contendo gelo reciclado. As amostras que apresentaram valores de creatinina inferiores a $0,3 \mathrm{~g} / \mathrm{L}$ ou superiores a $3,0 \mathrm{~g} / \mathrm{L}$ não foram consideradas. ${ }^{1}$ As urinas foram armazenadas, sem conservante, em nitrogênio líquido $\left(-130^{\circ} \mathrm{C}\right)$, até o momento da análise.

Após pequenas alterações, o método de Ducos et $\mathrm{al}^{5}$ (1990), modificado por Paula ${ }^{14}$ (2001), foi utilizado para a determinação do ATTM urinário.

A concentração do metabólito foi determinada por Cromatografia Líquida de Alta Eficiência com detecção ultra-violeta, empregando-se o método de quantificação por padronização externa, a partir de uma curva de calibração construída com soluções padrão de ATTM, em fase móvel, no intervalo de concentração de 0,03 a $4,0 \mu \mathrm{g} / \mathrm{mL}$.

A análise estatística foi realizada pelo software estatístico Sigma Stat for Windows 2.0, da Jandel Corporation $^{\circledR}$. Os valores de ATTM não apresentaram distribuição normal na população estudada. Portanto, o estudo estatístico foi executado empregando-se os testes não paramétricos de Kruskall-Wallis, Mann-Witney e de correlação de Spearman, todos eles com o critério de significância de $\mathrm{p}<0,05$.

\section{RESULTADOS}

\section{Grupo não exposto}

As amostras de urina do grupo não exposto apresentaram ATTM numa faixa de concentração variando entre $<0,03$ e $0,55 \mathrm{mg} / \mathrm{g}$ de creatinina, com valor médio de $0,10 \pm 0,08 \mu \mathrm{g} / \mathrm{g}$ de creatinina e mediana igual a $0,09 \mathrm{mg} / \mathrm{g}$ de creatinina.

Utilizando-se o critério proposto por Minoia et $\mathrm{al}^{12}$ (1990), a faixa de valor de referência do ATTM urinário na população analisada é de 0,03 a 0,26 $\mathrm{mg} / \mathrm{g}$ de creatinina. Esses dados estão de acordo com os relatados na literatura, onde foram encontrados valores basais variando de $<0,01$ a $0,66 \mathrm{mg} / \mathrm{g}$ de creatinina. ${ }^{3,6,9}$

Não foi encontrada a correlação entre ATTM urinário e sexo $(p>0,05)$, tampouco foi observada diferença significativa quando comparados os valores de ATTM nas amostras de indivíduos do sexo masculino e feminino.

Considerando o fato de a concentração do ATTM ter sido corrigida pela creatinina urinária e sabendose que este parâmetro urinário pode ser influenciado pelo gênero, os testes estatísticos foram repetidos, utilizando-se os valores de ATTM sem correção. A correlação entre o sexo e concentração do metabólito na urina continuou inexistente.

Foi demonstrado existir correlação positiva entre ATTM e idade $(\mathrm{p}<0,002)$, considerando-se a faixa etária como um todo. Ao subdividir-se a faixa etária em grupos (18 a 25; 26 a 35 e >36 anos), foi observada diferença significativa entre os valores de ATTM nos grupos de faixa etária de 18 a 25 anos e acima de 36 anos $(\mathrm{p}=0,016)$, conforme demonstrado na Tabela 2 .

Foi observada uma correlação positiva muito pequena entre ATTM e cigarro $(\mathrm{p}=0,048)$ no grupo de não expostos, mas o número médio de cigarros fuma-

Tabela 2 - Valores de ácido trans, trans-mucônico em amostras de urina de indivíduos não expostos ao benzeno, divididos de acordo com a faixa etária.

\begin{tabular}{lccc}
\hline Valores & \multicolumn{3}{c}{ ATTM (mg/g creatinina) } \\
& $\begin{array}{c}18 \text { a } 25 \text { anos } \\
(\mathrm{N}=39)\end{array}$ & $\begin{array}{c}26 \text { a } 35 \text { anos } \\
(\mathrm{N}=41)\end{array}$ & $\begin{array}{c}>36 \text { anos } \\
(\mathrm{N}=34)\end{array}$ \\
\hline Média $\pm \mathrm{IC}$ & $0,09 \pm 0,03$ & $0,09 \pm 0,02$ & $0,12 \pm 0,03$ \\
MG \pm DP & $0,05 \pm 0,10$ & $0,05 \pm 0,06$ & $0,09 \pm 0,08$ \\
Mediana & 0,06 & 0,09 & 0,10 \\
Valor mínimo & 0,03 & 0,03 & 0,03 \\
Valor máximo & 0,55 & 0,20 & 0,40 \\
\hline
\end{tabular}

ATTM - Ácido trans, trans-mucônico.

IC - intervalo de confiança a 95\%.

MG - média geométrica.

DP - desvio-padrão. 
dos por dia não demonstrou influenciar significativamente a concentração de ATTM.

Não se observou diferença estatística significativa $(\mathrm{p}=0,15)$ entre as concentrações médias de ATTM na urina de indivíduos fumantes e não fumantes.

Não foi encontrada a correlação $(p=0,84)$ entre o ATTM urinário e o hábito de ingerir bebidas alcóolicas na população de não expostos, assim como também não foi encontrada a diferença significativa entre os valores de ATTM, quando se comparou os indivíduos que haviam ingerido bebida alcóolicas àqueles que afirmaram não ter ingerido álcool.

Foi considerada ingestão alcóolica aquela ocorrida em um período menor ou igual a 48 horas antes da coleta da amostra biológica. Cinco indivíduos não especificaram o tempo decorrido entre a última ingestão de álcool e a hora da coleta e foram excluídos da análise. Esclarece-se que a amostragem do presente trabalho não foi delineada especificamente para o estudo da influência do álcool sobre a concentração do ATTM. Se assim fosse, seria necessário estabelecer a quantidade de bebida ingerida, o teor alcóolico desta bebida e um intervalo de tempo menor entre a ingestão e a colheita da amostra.

Considerando-se que o café é uma bebida consumida nacionalmente, avaliou-se se este hábito influenciaria a concentração urinária do metabólito. Os dados obtidos demonstraram não haver correlação entre a ingestão de café e a concentração de ATTM urinário.

\section{Grupo exposto}

Os níveis do ATTM urinário em amostras de urina de indivíduos expostos ao benzeno, coletadas ao final da jornada de trabalho, e as concentrações ambientais do solvente, obtidas ao nível da zona respiratória dos indivíduos durante as horas referentes ao respectivo turno de trabalho, encontram-se na Tabela 3.

Não foi observada a correlação significativa entre

Tabela 3 - Valores de benzeno no ar ocupacional e de ácido trans, trans-mucônico em amostras de urina dos indivíduos expostos ao solvente.

\begin{tabular}{lcc}
\hline Valores & $\begin{array}{c}\text { Benzeno no ar } \\
\left(\mathrm{mg} / \mathrm{m}^{3}\right)\end{array}$ & $\begin{array}{c}\text { ATTM urinário } \\
(\mathrm{mg} / \mathrm{g} \text { de creatinina })\end{array}$ \\
\hline $\mathrm{N}$ & 37 & 37 \\
Média $\pm \mathrm{IC}$ & $0,15 \pm 0,05$ & $0,19 \pm 0,04$ \\
$\mathrm{MG} \pm \mathrm{DP}$ & $0,16 \pm 0,16$ & $0,16 \pm 0,12$ \\
Mediana & 0,06 & 0,16 \\
Valor mínimo & 0,01 & 0,04 \\
Valor máximo & 0,94 & 0,67 \\
\hline
\end{tabular}

os níveis de ATTM na urina e os níveis de benzeno no $\operatorname{ar}(\mathrm{p}=0,26)$. Esse resultado pode ser explicado pelo baixo nível de exposição ocupacional encontrado no grupo exposto. O valor médio e mediana do benzeno no ar ocupacional foi $0,15 \mathrm{mg} / \mathrm{m}^{3}(0,05 \mathrm{ppm})$ e $0,06 \mathrm{mg} / \mathrm{m}^{3}(0,02 \mathrm{ppm})$, respectivamente.

Em relação às concentrações médias de ATTM urinário, foi encontrada a diferença significativa $(p<0,001)$ entre os indivíduos expostos e os não expostos ao benzeno.

Os valores de ATTM urinário nos indivíduos do grupo exposto foram também avaliados em função dos hábitos e características individuais dos componentes do grupo. Foi encontrada a correlação positiva com o hábito de fumar $(\mathrm{p}=0,0058)$ e diferença significativa entre o ATTM urinário nos indivíduos fumantes e não fumantes $(\mathrm{p}=0,008)$. No grupo de indivíduos não expostos ao benzeno, o cigarro não apresentou tão clara influência nos níveis urinários de ATTM.

O hábito de fumar influenciou também os níveis de benzeno no ar ocupacional $(p=0,034)$. Foi encontrada ainda a diferença significativa entre os níveis de benzeno nos grupos fumantes e não fumantes. A amostragem do benzeno no ar foi feita através de tubos coletores colocados à altura da zona respiratória do trabalhador, fato que levanta a hipótese do benzeno do cigarro que seria perdido com a fumaça não inalada estar sendo introduzido no organismo dos trabalhadores junto com o ar ocupacional respirado.

Não foi observada a correlação significativa entre o ATTM urinário e a faixa etária. Assim como o observado no grupo de não expostos, não foi encontrada a correlação entre ATTM urinário e ingestão de bebidas alcóolicas e café.

\section{DISCUSSÃO}

Os valores de ATTM encontrados nas amostras de urina do grupo não exposto (entre $<0,03$ e $0,55 \mathrm{mg} / \mathrm{g}$ de creatinina) estão de acordo com os relatados na literatura, onde foram encontrados valores basais variando de $<0,01$ a $0,66 \mathrm{mg} / \mathrm{g}$ de creatinina. ${ }^{3,6,9}$

Os resultados deste trabalho mostram que o ATTM não apresenta sensibilidade suficiente para correlacionar-se com níveis de benzeno no ar tão baixos quanto os encontrados no presente estudo (média de $0,15 \pm 0,05 \mathrm{mg} / \mathrm{m}^{3}$ ou $0,05 \mathrm{ppm}$ ). A ausência de correlação observada está de acordo com os dados da literatura que demonstraram sensibilidade 
do ATTM urinário apenas para detectar exposições ao benzeno em níveis superiores a $0,1 \mathrm{ppm} .{ }^{10,11}$

Este biomarcador é, entretanto, bastante sensível para diferenciar expostos de não expostos, mesmo em exposições tão baixas quanto a encontrada no presente trabalho.

A influência do hábito de fumar nos níveis de ATTM urinário dos indivíduos expostos e a diferença significativa encontrada entre as concentrações do metabólito na urina de fumantes e não fumantes são dados importantes no estudo de validação do ATTM como biomarcador de exposição ocupacional ao benzeno. Entretanto, considerando o número de amostras de expostos disponível para o estudo (13 fumantes e 24 não fumantes), torna-se necessário confirmar estes resultados ampliando-se a amostragem de expostos fumantes e não fumantes.

Os dados obtidos na análise do grupo de indivíduos não expostos não permitem estabelecer, com confiança, a influência do hábito de fumar sobre os níveis de ATTM nestes indivíduos. Na literatura, alguns autores relatam ser o hábito de fumar um fator interferente, que contribui para diferenças significativas nos níveis urinários de ATTM. ${ }^{4,8}$ Outros afirmam não ter detectado qualquer correlação entre cigarro e ATTM urinário. ${ }^{5,11} \mathrm{O}$ pequeno número de indivíduos fumantes dentro do grupo de não expostos $(\mathrm{N}=22)$ pode ter dificultado uma melhor interpretação dos dados analíticos obtidos. Estudos em populações maiores deverão ser realizados a fim de se comprovar a influência do cigarro sobre a concentração urinária de ATTM.

A concentração do ATTM na urina de indivíduos expostos ao benzeno não demonstrou influência da faixa etária. Este dado contrapõe-se àqueles relatados na análise do grupo de não expostos, na qual foi observada diferença significativa entre os níveis de ATTM na faixa etária de 15 a 26 anos e acima de 36 anos. Observou-se que a média de idade dos indivíduos do grupo não exposto foi de 30,7 anos (mediana de 29 anos) e a do grupo de expostos 34,8 anos (mediana de 36 anos). Esta diferença poderia ser a explicação para a não correlação entre idade e ATTM no grupo exposto. No entanto, uma hipótese como a levantada acima necessita de estudos mais amplos para ser confirmada.

\section{REFERÊNCIAS}

1. Alessio L, Berlin A, Toffoletto F, Ghezzi I. Reability of urinary creatinine as a parameter used to adjust of urinary biological indicators. Int Arch Occup Environ Health 1985;55:99-106.

2. Barbosa EM. Exposição ocupacional ao benzeno: o ácido trans, trans-mucônico como indicador biológico de exposição na indústria de refino de petróleo. Rio de Janeiro: Petrobrás/Serviços de Recursos Humanos; 1999. p. 1-48.

3. Boogaard PJ, Van Sitter NJ. Biological monitoring of exposure benzene: a comparison between $\mathrm{S}$ phenylmercapturic acid, trans, trans-muconic acid and phenol. Occup Environ Med 1995;52:611-20.

4. Buratti M, Fustinoni S, Colombi A. Fast liquid chromatography determination of urinary trans, transmuconic acid. J Chromatogr B 1996;677:257-63.

5. Ducos P, Gaudin R, Robert A, Francin JM, Meire C. Improvement in HPLC analysis of urinary trans, transmuconic acid, a promissing substitute for phenol in assessment of benzene exposure. Int Arch Occup Environ Health 1990;62:529-34.
6. Ducos P, Gaudin R, Bel J, Marie C, Francin JM, Robert A et al. Trans, trans- muconic acid, a reliable biological indicator for the detection of individual benzene exposure down to the ppm level. Int Arch Occup Environ Health 1992;64:309-13.

7. Freitas NBB, Arcuri ASA. Valor de referência tecnológico (VRT)- a nova abordagem do controle da concentração de benzeno nos ambientes de trabalho. Rev Bras Saúde Ocup 1998;24:71-85.

8. Ghittori S, Maestri L, Fiorentino M, Imbriam M. Evaluation of occupational exposure to benzene urinalysis. Int Arch Occup Environ Health 1995;67:195-200.

9. Gobba F, Rovesti S, Borella P, Vivoli R, Caselgrandi E, Vivoli G. Inter-individual variability of benzene metabolism to trans, trans-muconic acid and its implications in the biological monitoring of ocupacional exposure. Sci Total Environ 1997;199:41-8.

10. Kivistö H, Pekari K, Peltonen K, Svinhufvud J, Veidebaum T, Sorsa $M$, et al. Biological monitoring of exposure to benzene in the production of benzene and in a cokery. Sci Total Environ 1997;199:49-63. 
11. Lee BL, New AL, Kok PW, Ong HY, Shi $C Y$, Ong $C N$. Urinary trans, trans-muconic acid determined by liquid chromatography: application in biological monitoring of benzene exposure. Clin Chem 1993;39:1788-92.

12. Minoia C, Sabbione E, Apostoli P, Pietra R, Pozzoli L, Gallorini $M$, et al. Trace element reference values in tissues from inhabitants of the european community I. A study of 46 elements in urine, blood and serum of italian subjects. Sci Total Environ 1990;95:89-105.

13. Ong CN, Kok PW, Lee BI, Shi CY, Ong HY, Chia KS et al. Evaluation of biomarkers for occupational exposure to benzene. Occup Environ Med 1995;52:528-33.
14. Paula FCS. Validação do ácido trans, trans-mucônico como biomarcador de exposição ao benzeno [dissertação]. Belo horizonte (MG): Universidade Federal de Minas Gerais; 2001.

15. Pezzagno G, Maestri L, Fiorentino ML. Trans, transmuconic acid, a biological indicator to low levels of environmental benzene: some aspects of its specificity. Am J Ind Med 1999;35:511-8.

16. Scherer G, Renner T, Meger M. Analysis and evaluation of trans, trans-muconic acid as a biomarker for benzene exposure. J Cromatogr B 1998;717:179-99. 